Faculdade de Ciências Econômicas UFRGS
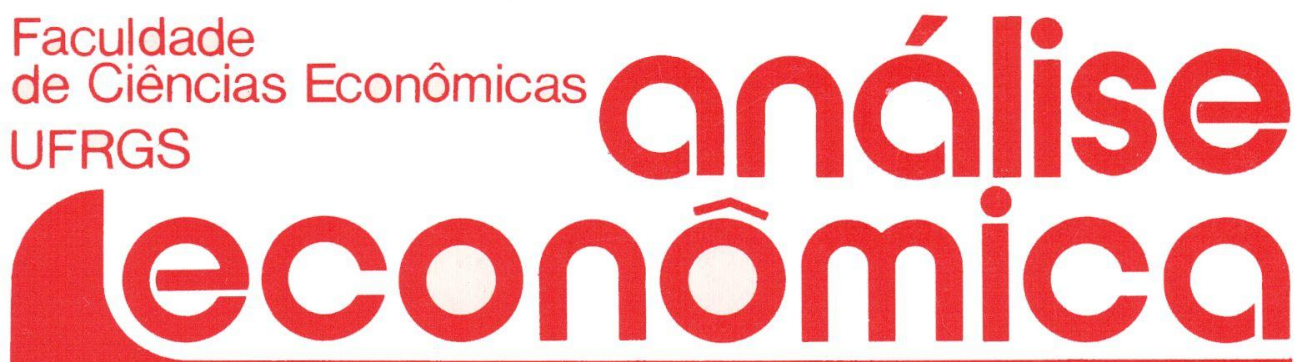

nesta edição:

- PIERO SRAFFA: 1898 - 1983

Nicholas Kaldor

- A BUSCA DOS FUNDAMENTOS, SEM CHOQUES

Yeda Rorato Crusius

- estado e acumulação DO CAPITAL

Paulo Nakatani

- abastecimento

ALIMENTAR

BRASILEIRO

Edgar Irio Simm

- política

RIOGRANDENSE NA REPÚBLICA VELHA

Céli Regina J. Pinto
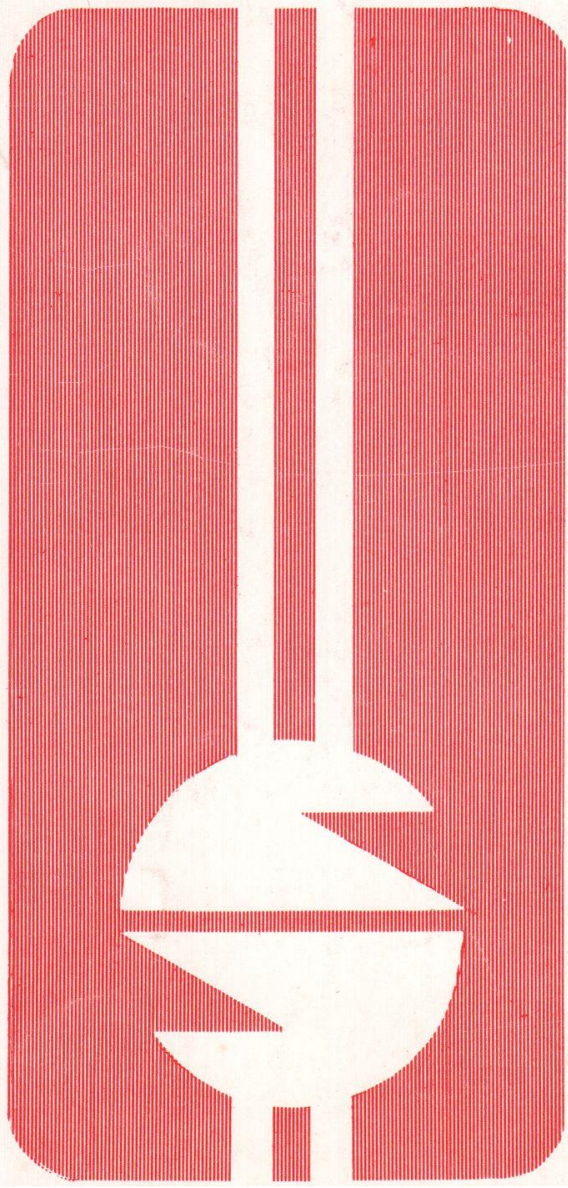
REITOR: Prof. Francisco Ferraz

DIRETOR DA FACULDADE DE CIENCIAS ECONOMICAS:

Prof. Edgar Irio Simm

VICE-DIRETOR: Prof. Walter Meucci Nique

CHEFE DO DEPARTAMENTO DE CIÉNCIAS ECONOMICAS:

Prof. Ernani Hickmann

CONSELHO EDITORIAL:

$$
\begin{aligned}
& \text { Prof. Pedro Cezar Dutra Fonseca (Presidente) } \\
& \text { Prof. Achyles Barcelos da Costa } \\
& \text { Prof. Carlos Augusto Crusius } \\
& \text { Prof. Claudio Francisco Accurso } \\
& \text { Prof. Edgar Augusto Lanzer } \\
& \text { Prof. Ernani Hickmann } \\
& \text { Prof. Juvir Mattuella } \\
& \text { Prof. João Rogério Sanson } \\
& \text { Prof. Maria Imilda da Costa e Silva } \\
& \text { Prof. Nali de Jesus de Souza } \\
& \text { Prof. Nuno Renan L. de Figueiredo Pinto } \\
& \text { Profa Otilia Beatriz Kroeff Carrion } \\
& \text { Profa Yeda Rorato Crusius } \\
& \text { Prof. Paulo Alexandre Sphor } \\
& \text { Prof. Roberto Camps Moraes }
\end{aligned}
$$

FUNDADOR:

$$
\text { Prof. Antonio Carlos Rosa }
$$

ANÁLISE ECONÓMICA publica dois números anuais nos meses de março e novembro. O preço da assinatura para 1987 é $\mathrm{Cz} \$ 60,00$, a ser pago através de cheque nominal para "Faculdade de Ciências Econômicas - UFRGS". Aceita-se permuta com revistas congêneres. Aceitam-se, também, livros para elaboração de resenhas ou recensões.

Toda a correspondência, material para publicação, assinaturas e permutas devem ser dirigidas a:

Prof. PEDRO CEZAR DUTRA FONSECA

Revista Análise Econômica

Av. João Pessoa, 52 - 3. andar

90.000 - Porto Alegre (RS) - Brasil 


\title{
A BUSCA DOS FUNDAMENTOS, SEM CHOQUES
}

\author{
YEDA RORATO CRUSIUS *
}

\section{INTRODUÇÃO:}

O que terá dado errado? Esta parece estar sendo a questão mais freqüentemente levantada entre os estudiosos e aplicadores de política econômica da atualidade. Afinal, os resultados das experiências recentes que tentam controlar os movimentos cíclicos como esses se apresentam no moderno capitalismo financeiro não têm sido reconfortantes. A busca pelos princípios da teoria macroeconômica que permitam propor linhas de ação para controle do ciclo tem orientado o fermentar intelectual desde que passaram a conviver tão intensamente recessão com inflação. Em particular, a evolução recente das economias em desenvolvimento tem se caracterizado por um persistente incremento da instabilidade e pela agudização das situações de desequilibrio, causadas pela (aparente) irreversibilidade de seus processos inflacionários e pelos desajustes estruturais de seus balanços de pagamentos, devido à particular inserção dessas economias no cenário internacional. Busca-se identificar o que, nesta trilha de instabilidade, é fruto dos movimentos cíclicos peculiares do desenvolvimento capitalista, o que decorre de diagnósticos e de modelos que resultam na aplicação de politicas econômicas equivocadas.

Os desafios analiticos e as dificuldades presentes no caminho tentativo dessa identificação são de distintas naturezas. Dentre eles, e o que importa para o caso neste texto, está o reconhecimento de que as relações de causalidade em um contexto de instabilidade possuem contornos imprecisos, ampliados pelas constantes alterações nos campos político e institucional nas economias em desenvolvimento, assim como pela aplicação de políticas ainda mais desestabilizantes do que as do tipo "stop-and-go" do receituário descendentes do Plano

* Professora do Departamento de Ciências Econômicas e do Curso de Pós-Graduação em Economia da Universidade Federal do Rio Grande do Sul.

\begin{tabular}{|l|l|l|l|l|}
\hline ANÁLISE ECONÔMICA & ANO 5 & N. 8 & MARÇO/87 & p. 25-34 \\
\hline
\end{tabular}


Marshall e do Sistema Financeiro Internacional estruturado após a II Grande Guerra.

No campo teórico, o Movimento que sacudiu o pensamento económico trazido pela Teoria Geral de Keynes, publicada em 1936, terminou por desestruturar o arcabouço da teoria dos "clássicos", como o próprio Keynes rotulou alguns dos pensadores econômicos relevantes daquela época. O desenvolvimento das idéias relativas a equilíbrio macroeconómico, método e ação, contidas na Teoria Geral, levou à reação pela necessidade de restabelecimento dos principios da escola neoclássica, como sintetizado na parte $\mid$ do presente texto. Fraturando o princípio neoclássico de agregação de comportamento individual considerado para explicação dos fenômenos econômicos, através da especificação do "paradoxo da composição", Keynes jogou luz a um claro metodológico que tenta ser preenchido através dos estudiosos dos fundamentos microeconômicos da macroeconomia (SIMON, 1956, MUTH, 1960, HICKS, 1974, WEINTRAUB, 1979). A parte II deste texto trata de apresentar algumas das discussões suscitadas através desta linha de ataque ao problema da compatibilização entre as duas áreas da ciência econômica. A discussão apontada oferece a oportunidade do levantamento de questões, bem mais do que conclusões, na parte III que finaliza este artigo.

\section{I - O PENSAMENTO CONTEMPORÂNEO DA ECONOMIA NEOCLÁSSICA}

A Revolução trazida por Keynes através da Teoria Geral, bem como os desenvolvimentos posteriores que dão a moldura à chamada teoria keynesiana, partem da crítica aos pressupostos "clássicos" como analisados pela Teoria Geral, e aqui transcritos resumida e livremente da forma que segue:

1. O sistema econômico se resume num conjunto de trocas em mercados independentes as quais, a partir do comportamento do agenté individual, podem ser agregadas pela soma resultando no todo macroeconômico. Nesse sistema, a moeda $(M)$ tem as funções de unidade de conta e reserva de valor para trocas futuras, não sendo procurada por ela própria. A moeda é apenas o "véu" do sistema de trocas, o denominador comum"para cálculo do valor. $A$ taxa de juros não representa o preço da moeda, e sim a taxa que, na esfera agregada, leva a que os mercados de bens (investimento e poupança) se equilibrem através de um "preço natural", ou taxa "natural" de juros. Essa taxa elimina do mercado os excessos de investimento ou de poupança derivados da discrepância entre plano e realização. O sacrificio ao con- 
sumo (poupança) se igualará à rentabilidade das oportunidades de investimento: o mercado de bens determinará os juros, que são um preço que equilibra duas quantidades REAIS.

2. O mercado de moeda é representado pela teoria quantitativa da moeda através da qual, dado o nivel de produto, fica determinado o nivel geral de preços $(P)$, e não qualquer preço relativo. $P$ é o nível de preços absoluto e, respeitado o produto de pleno-emprego, é determinado pela quantidade de moeda do sistema. Variações em $P$ não causam alterações na distribuição (mudança de relativos) sendo, portanto, a moeda neutra.

3. Se deixada funcionar livremente - o sistema do "laissez-faire" - a economia resultará em um sistema de eficiência e bem-estar máximos. As duas condições que precisam ser preenchidas para essa equilíbrio AUTOMÁTICO E DE PLENO EMPREGO são a plena flexibilidade de todos os preços e o comportamento racional de cada e de todos os agentes econômicos. A plena flexibilidade assegura que, frente a desequilíbrios, a variação de preços eliminará em cada mercado, e em todos simultaneamente, qualquer excesso de oferta ou de demanda. As figuras do leiloeiro walrasiano e do mercado de peixes marshaliano ilustram esse processo de ajustamento automático a desequilibrios através de preços (MUELLER, 1983). A racionalidade pelo preenchimento das condições de maximização em ambiente de plena informação asseguram, por sua parte, o funcionamento da "lei" de Say: o ser econômico "racional" (maximizador) produzirá sempre o máximo (oferta de pleno-emprego) e criará a sua própria demanda (uma vez aceita plena flexibilidade dos preços por parte de quem oferece e de quem procura).

4. Em qualquer mercado, o valor é determinado pelo sacrifício realizado para a obtenção da unidade marginal, ou seja, pelo preço. O preço do trabalho, como o de qualquer outro produto ou serviço, é determinado pelo jogo da oferta e da procura sob condições de maximização pela margem. Havendo excesso de oferta de trabalho, seu preço deverá baixar até que se atinja o equilíbrio no emprego da força de trabaIho.

5. Os ciclos econômicos são, portanto, distanciamentos fortuitos da norma de funcionamento do sistema econômico clássico, e derivam de desequilibrios internos de causas monetárias ou bancárias ( $M$ ou i inadequadas) ou de desequilibrios temporários nas trocas internacionais, corrigiveis por politica carnbial/monetária adequada tendo como guia a teoria das vantagens comparativas de David Ricardo.

Através da crítica a essas hipóteses "clássicas", Keynes formulou um sistema no qual a taxa de juros é preponderantemente um fenô- 
meno monetário determinado pela preferência pela liquidez e que, através da. função-investimento, torna o mercado de moeda e o de bens, baseados em relações de comportamento macroeconômico, independentes. Se equilibrados ambos, conforme sistematizado por Hicks através do modelo IS/LM, fica determinado o produto demandado (Yd). A demanda efetiva; entretanto, é "o emprego que os empresários estão dispostos a contratar" (KEYNES, 1936) frente a um determinado nivel de demanda agrègada. Em termos comuns, é o cruzamento entre a oferta e a demanda agregadas. O mercado de fatores, então, determinará o nivel de demanda efetiva. $O$ equilíbrio, neste mercado, depende da relação entre custos e receitas (W/P). A ligação entre os mercados monetário, de bens e de fatores, se faz através de cada possível par (i,P), determinando-se assim o nivel de produto e de emprego.

Para o mercado de fatores, entretanto, Keynes parte das hipóteses neoclássicas com base na receita de maximização pela margem, a partir de funções "bem comportadas", sendo que a agregação para se chegar a Y é a mesma dessa escola: é a soma dos comportamentos individuais. A presença de expectativas na determinação da oferta e a independência entre os mercados, entretanto, é que levam à conclusão de Keynes de que é possivel (e provável) o equilibrio entre os três mercados na presença de desemprego. As principais fontes do desequilibrio (desemprego) na forma de inconsistências possiveis apontadas por ele, são (a) a inflexibilidade de salários e preços face à presença de sindicatos e de fatores de concentração de mercados, com ênfase nos primeiros; (b) a armadilha para a liquidez; (c) a "ilusão monetária"; (d) a presença de expectativas e incertezas nas funções relevantes.

As questões suscitadas pela Teoria Geral tanto no campo macroeconômico, no qual os pressupostos de determinação de $\mathrm{P}$, de $\mathrm{Ye}$ de i em mercados independentes são desmontados, quanto no campo microeconômico, no qual as hipóteses de racionalidade e de plena flexibilidade de preços ficam sob juízo, abalam a estrutura montada sobre a simplicidade e a excelência matemática do sistema de equilíbrio geral neoclássico. E principalmente à partir dos anos 50 que as discussões sobre os fundamentos tanto da direção da causalidade em economia (HICKS, 1979) quanto da racionalidade nas decisões econômicas (MUTH, 1961 e SIMON, 1956) se aprofundam. A questão não se restringe ao campo teórico, senão que se estende à preocupação de lastro para a tomada de decisões de política econômica, modernamente intensificada face à presença de sucessivas crises financeiras (MINSKY, 1982).

Conforme aponta a literatura atualmente disponivel no pais, in- 
centivada pelos eventos comemorativos dos 100 anos de Marx (morte) e Keynes (nascimento), bem como os 50 anos da TEORIA GERAL (ver, por exemplo, as idéias de diversos autores publicadas nos Anais dos Encontros da ANPEC - Associação dos Centros de Pós-graduação em Economia, e na revista PESQUISA E PLANEJAMENTO ECONÔMICO de outubro de 1986), o pensamento neoclássico passa a elaborar sua sintese em resposta aos desenvolvimentos pós-keynesianos nos campos micro e macroeconômicos, fundamentada nas seguintes hipóteses:

(1) a racionalidade do agente econômico fica reestabelecida dentro dos parâmetros neoclássicos ao se ampliar a noção de escolha através da aceitação do processo de formação das expectativas racionais. Os agentes econômicos, ao formularem suas decisões (subjetivas) consideram os PROVÁVEIS valores das variáveis que influenciam suas decisões, dada a incerteza sobre o futuro; o referencial objetivo (a realidade) fornece a base para esse agente determinar os valores esperados dos fenômenos que importam. Partindo do pioneiro trabalho elaborado por MUTH, em 1961, iniciaram-se os estudos que buscaram avaliar quantitativamente os processos de hiperinflação e de desequilíbrio no setor agrícola através de um método onde se possibilita a interação entre as expectativas e a realidade (expectativas condicionadas), bem como a dinâmica de formação do comportamento individual com base em predição dos acontecimentos futuros (função-demanda), determinação do preço por parte das empresas, escolha de portfolio, etc.). A própria neutralidade da moeda fica restaurada através do comportamento racional na dinâmica de curto prazo. O comportamento do consumidor é redesenhado considerando ser o mesmo fruto de expectativas racionais e sujeito, primordialmente, aos cálculos racionais referenciados pelo custo presente do sacrifício da melhor alternativa, ou, pela taxa de juros. O agregado é a soma dos comportamentos individuais assim estabelecidos, o qual necessita, no campo macroeconômico, de informações corretas para formular sua decisão dinâmica. Exemplo desta vertente é o da teoria aceleracionista de Friedman, na qual os desequilíbrios de relativos são eliminados quando os contratos são realizados com base na expectativa de inflação. Por esta tese, o "trade-off" presente nas análises a la Phillips desaparece no longo prazo, negando os fundamentos ativistas para a ação através de políticas de controle do ciclo da tradição Keynesiana.

(2) o ajustamento via preços, possivel dentro dos mercados walrasiano (através do leiloeiro) ou marshalliano (mercado de peixes) é restaurado pela aceitação de que se contrata em mercados futuros, e não apenas em mercados correntes. $O$ ajustamento via preços é fruto das deci- 
sões, agora dinamizadas, dos agentes econômicos, reeditando-se o vetor de preços do equilíbrio geral.

(3) as fontes básicas de incerteza na esfera macroeconômica, que geram desequilíbrios a nível de decisão individual, devem ser eliminadas pelo estabelecimento de regras fixas de política (em lugar de políticas de ajustamento ao ciclo do tipo "fine-tuning") especialmente no campo monetário. Neste caso, a paternidade das flutuações cíclicas recaem explicitamente sobre a atuação do "governo ativista, de inspiração keynesiana". Adicionalmente, a intervenção através do estabelecimento de cláusulas contratuais de proteção contra variações monetárias futuras, como as cláusulas de indexação, devem ser estabelecidas para reduzir ou anular incertezas em relação ao futuro. Elevações de P, neste caso, voltam a ser neutras.

(4) finalmente, os desajustes macroeconômicos podem ser eliminados ao se seguir a regra de remuneração pela produtividade marginal. $O$ equilibrio entre poupança e investimento é feito pela "regra de ouro da acumulação", que restaura o pleno-emprego com eficiência num contexto dinâmico de crescimento.

Reconhecendo a presença de fatores que distanciam do sistema de concorrência perfeita o moderno capitalismo, como externalidades, empresas gigantes, ação de oligopólios, e instabilidade do comércio internacional (derivada tanto dos planos de desenvolvimento do Sul quanto ao neoprotecionismo do Norte, que distorcem o funcionamento do "livre comércio", e da ausência do referencial do padrão-ouro), os economistas neoclássicos contemporâneos aceitam a interferência ativa do governo o qual, através da imposição de impostos, tarifas e subsídios, pode levar à alocação eficiente dos recursos e à correção das distorções de distribuição (mesmo que a nivel de "segundo melhor"). $\mathrm{O}$ "laissez-faire", embora não restaurado como realidade e possibilidade pois que "qualificado", permanece à base do paradigma neoclássico, e o seu ideal.

Finalmente, desajustes (cíclicos) do balanço de pagamentos devem ser tratados através de política cambial e monetária,consubstanciadas no enfoque monetário do balanço de pagamento. Em suma, para a síntese neoclássica moderna, a aceitação dos desequilibrios a nível de um mercado, ou de multimercados, ou macroeconômico, são explicados apenas pela quebra das pressuposições do equilíbrio geral em concorrência perfeita com ajustamento instantâneo. Substituindo o leiloeiro walrasiano por um sistema de informaçōes adequado à contratação a futuro, guiado pelo "governo" neoclássico contemporâneo, ficam restabelecidas as condições de equilibrio através da formação das expectativas racionais. 
Dentro da tradição keynesiana, entretanto, existe a dicotomia de fundamentos: os mercados de RELAÇÕES CAUSAIS AGREGADAS são os mercados monetário (LM) e de bens (IS) que determinam a demanda agregada. A oferta agregada, entretanto, parte de relações microeconômicas e aceita a "agregação pela soma", na qual não cabe o paradoxo da composição presente na função consumo, base da IS, ou o "estado geral das expectativas", representado pela armadilha pela liquidez.. Os preços (ou P, o nivel geral de preços) são formados fora dos mercados IS/LM, embora os influenciem deterministicamente para cada dada distribuição de renda, ou pela consideração do efeito riqueza-real na própria função-consumo. O nivel geral de preços é determinado nos mercados de fatores, e partir de um certo "estado de expectativas" por parte dos empresários que formulam os seus planos reais de produção, ou seja, de oferta.

$A$ equação de $P$ para Keynes inclui os elementos de custo, de planta, de expectativas quanto à evolução a fase do ciclo, como principais. Para uma determinada estrutura econômica, a fase do ciclo (capacidade ociosa), o processo de formação da taxa de salários monetários média (contratos pela taxa nominal, que permite a existência de "ilusão monetária"), e o estado geral das expectativas - todos eles fatores macroeconômicos - conduzem a evolução do nivel geral de preços $P$. Os elementos micro (custos de uso, salários e comportamento individual pela soma) e macro considerados por Keynes, entretanto, são apenas a introdução para o problema da dicotomia entre preços relativos e preços absolutos. A dinâmica da formação dos lucros sob o esquema departamental de KALENCKI (1956) é fundamental para o estudo neste campo. Para o que nos interessa, PHILLIPS (1958) e BACHA (1984, como exemplo) podem ser considerados como propositores seminais de questões de ação prática, ou ação política, a partir da aceitação de uma equação de $P$ determinada na preseça de hiatos de recursos, tanto internos quanto externos. Conforme os estudos que se seguem às pistas plantadas por estes dois autores, o desequilíbrio macroeconômico implica em escolhas de política substancialmente diferentes das comumente sugeridas por padrões internacionais. $O$ avanço em termos de formulação de modelos e de propostas de estabilização (a "escolha" entre inflação e desemprego) é notável na esteira do desenvolvimento das idéias de ambos os autores citados (ver SIMONSEN, 1983, e TAYLOR, 1982).

Os fundamentos para essa ação, entretanto, levam à pesquisa 
adicional a respeito das decisões a nívęl microeconômico, como exposto tanto pelos estudos da Incerteza e Risco quanto pela busca das razões que levam à fixação dos preços por parte dos agentes individuais (empresas). De todas as maneiras, o desenvolvimento do campo pertencente à influência da estrutura (relativos) na determinação de $P$, e o dos estudos das expectativas racionais têm influenciadó, sem dúvida, o entendimento da macroeconomia moderna. $E$, portanto, relevante para a compreensão dos fenômenos agregados e para a escolha de políticas de controle do ciclo.

\section{III - ERROS, APRENDIZADO E JULGAMENTO}

Dado o estágio incompleto de desenvolvimento das investigações a respeito dos fundamentos microeconômicos da macroeconomia, não se deve estranhar como tantos economistas de peso, nome e responsabilidade tenham "apostado" numa linha de politica de combate à inflação baseada na doma das expectativas e dos preços de mercado, como a que foi elaborada e aplicada através de "choques heterodoxos". A pergunta que se impõe fazer por parte de todos os que foram responsáveis, de algum modo, pela experiência que lançou o país num retrocesso econômico similar à experiência da recessão programada à la FMI, da qual buscava-se escapar que no campo teórico, quer no aplicado, é O QUE TERÁ DADO ERRADO (INFORMAÇŌES FIPE, NNo 82, fev/87).

A resposta não terá sido completa sem que se busque pelos princípios, sem choques: como são determinadas as decisões, a_nivel de agente microeconômico, e como se dá o processo de decisão à nível de relações agregadas. A busca é pelos fundamentos microeconômicos da macroeconomia, que são parte do desconhecimento à respeito das conseqüências da aplicação de políticas de controle da demanda como as de tradição keynesiana num contexto de instabilidade com concentração (Norte/Sul, setorial, tecnológica). A crescente ineficácia na aplicação de políticas monetárias e fiscais, de cunho nitidamente agregado, como instrmento de controle das flutuações, esbarra no desconhecimento de forma e alcance de suas alternativas, que são as políticas de controle dos processos de formação das expectativas e dos preços relativos.

Temos sido levados a avaliar o atual estágio de desenvolvimento das idéias neste campo através (a) da discussão acerca do método, como sintetizado em MUELLER (1983), ou (b) da ação através de controles diretos, dentro da linha analítica da moderna análise estruturalista, ou (c) das causas que possivelmente podem nos levar a novas e 
gigantescas crises financeiras (ciclos financeiros) à la Minsky. As linhas de investigação, quaisquer que sejam reconhecem o processo real (objetivo) de crescente instabilidade econômica, bem como a incompletude teórica no que tange à integração sistemática entre as duas esferas da ciência econômica, a micro e a macro. Apesar disto, as decisões econômicas, mesmo que baseadas em evidências imperfeitas, continuam a ser tomadas. Com base em probabilidades, os julgamentos são feitos, e as ações implementadas. O desenvolvimento na busca de fundamentos poderia, através da avaliação dos erros de diagnóstico e de aplicação, no confronto entre o possível e o provável, orientar para escolhas politicas que minimizem os custos dinâmicos das experiências.

\section{BIBLIOGRAFIA SELECIONADA}

1. BACHA, E. Crescimento e Oferta Limitada de Divisas: uma Reavaliação do Modelo dos Dois Hiatos. PESQUISA E PLANEJAMENTO ECONOMICO, 12(2), Ag. 1982.

2. BACHA, E. e ARIDA, P. Balanço de Pagamentos: uma Análise de Desequilíbrio para uma Economia Semi-industrializada. PESQUISA E PLANEJAMENTO ECONÓMICO, 14 (1), Abr. 1984.

3. BACHA, E. Choques Extern is e Perspectivas de Crescimento: O Caso do Brasil. PESQUISA E PLANEJAMENTO ECONOMICO, 14(4), Dez 1984.

4. BARRO, R.J. Unantecipated Monetary Growth and Unemployment in U.S. AMERICAN ECONOMIC REVIEW, n.67, 1977.

5. CASTRO, A.B. Keynes e a Velha Tradição do Ciclo. PESQUISA E PLANEJAMENTO ECONOMMICO, 16(2), Ag 1986.

6. CLOWER, R.W. The Keynesian Counterrevolution: a Theoretical Appraisal em HAHN \& BRECHLINGS (Eds), Macmillan, 1965.

7. CRUSIUS, Y.R. A (Há?) Escolha entre Inflação e Desemprego. PERSPECTIVA ECONO̊MICA, UNISINOS, 11(34), 1981.

8. CRUSIUS, Y.R. A Relação entre Salário e Desemprego. TEXTO DIDÁTICO CPEGE/UFRGS N.01, 1986. 
9. FRENKEL, R. Salários e Inflação na Arnérica Latina: Resultados das Pesquisas Recentes na Argentina, Brasil, Chile, Colômbia e Costa Rica. PESQUISA E PLANEJAMENTO ECONÓMICO, 16(1), Abr 1986.

10. HICKS, J. CAUSALITY IN ECONOMICS. Basic Books, 1979.

11. KALECKI, M. TEORIA DA DINÅMICA ECONOMICA. OS Pensadores, Coleção Abril, 1956.

12. KEYNES, J.M. TEORIA GERAL DOJURO, DO EMPREGO E DO DINHEIRO. Os Economistas. Série da Abril Cultural, 1984 (1936).

13. MINSKY, H.P. CAN IT HAPPEN AGAIN? ESSAYS IN INSTABILITY AND FINANCE. NEW YORK, M.E. SHARPE INC., 1982.

14. MUELLER, A.E.G. Macroeconomia do Desequilíbrio e Post-keynesianismo frente ao Equilíbrio Geral: Algumas Reflexões Comparativas. ANAIS DO XI ENCONTRO DA ANPEC, 1983.

15. MUTH, J.F. Rational Expectations and The Theory of Price Movements. ECONOMETRICA, 29(3), Jul 1961.

16. PHILLIPS, A.W. The Relation between Unemployment and the Rate of Change in Money Wage Rates in U.K. - 1981/ 1957. READINGS IN MACROECONOMICS, Ed. MUELLER, 1958.

17. SARGENT, T. Rational Expectations, the Real Rate of Interest and the Natural Rate of Unemployment.

18. SIMONSEN, M.H. DINAMICA ECONÓMICA. São Paulo, MCGRAW-HILL, 1983.

19. . Keynes vs Expectativas Racionais. PESQUISA E PLANEJAMENTO ECONOMICO, 16(2), Ag 1986.

20. WEINTRAUB, E.R. MICROFOUNDATIONS OF MACROECONOMICS. CAMBRIDGE UNIVERSITY PRESS, 1979. 